\title{
Aplicação das Técnicas de Projeto Instrucional 4C/ID na Produção de Objetos de Aprendizagem em Conformidade com o SCORM Usando um Software Livre como Ferramenta de Autoria
}

\author{
Adilson Vahldick ${ }^{1}$ \\ Rafael de Santiago ${ }^{1}$ \\ André Luís Alice Raabe ${ }^{1}$ \\ ${ }^{1}$ Mestrado em Computação Aplicada - Universidade do Vale do Itajaí (UNIVALI) \\ adilsonv77@gmail.com, \{rsantiago, raabe\}@univali.br
}

Resumo: Esse artigo apresenta um estudo de caso de confecção de material instrucional para um curso ilustrando as possibilidades de sequenciamento das atividades com a especificação SCORM. Para autoria desse curso foi utilizada a ferramenta MOS Solo, e baseado no método 4C/ID para o projeto instrucional do conteúdo.

Palavras-chave: projeto instrucional, scorm, 4c/id, mos solo, autoria de objetos de aprendizagem.

Abstract: This works presents a case study where an instructional content of a course is created ilustrating the possibilities of activities sequencing with the SCORM model. For authoring of this course was used the MOS Solo software and based on the 4C/ID method to the instrucional design of the content.

Keywords: instructional design, scorm, 4c/id, mos solo, learning objects authoring

\section{Introdução}

Para a disseminação no uso de tecnologias na educação tem se defendido a formação dos professores para a produção de conteúdo através de ferramentas de autoria, como o Flash, Power Point e Hot Potatoes (Tarouco et al, 2006). Entretanto, o conhecimento no uso das ferramentas não é garantia para que o material consiga atender os objetivos de aprendizagem. É preciso refletir como estruturar o material e em que momento ele deve ser entregue ao aluno.

Em uma instituição de ensino, a adoção de uma metodologia pelos professores para a produção desse material ajuda na melhoria da qualidade, pois o uso contínuo e comum na produção do material acarreta no amadurecimento e melhoria da própria metodologia. Existem várias metodologias publicadas, que são denominadas projeto instrucional. Nesse artigo será apresentada uma delas chamada de 4C/ID, onde é sugerida uma estrutura de aula dividida em quatro componentes integrados. Esse modelo tem um divisão bem definida de como uma aula deve ser estruturada, e a existe a preocupação de diminuir a carga cognitiva para o aluno, ou seja, fazer com que ele assimile gradativamente.

Com a popularização da informática nas instituições de ensino, cada vez mais professores se arriscam a produzir material digital para suas aulas. A disponibilização desse material aos alunos ocorre através de um ambiente de aprendizagem (AVA). Esses ambientes possuem recursos como fórum, chats e área para postagem de material. Contudo, outro tipo de sistema na área educacional já é consagrado no exterior, que é o Learning Management System (LMS). Esse tipo de sistema, possui todos os recursos de um AVA, e além disso, acompanha o desempenho do aluno e pode fornecer o material de acordo com as necessidades de cada um (Irlbeck e Mowat, 2007). Mas para que o 
LMS consiga executar essas tarefas o material precisa ser produzindo segundo alguns critérios e estruturas.

Para que o material produzido para executar no LMS de um fabricante possa ser executado no LMS de outro fabricante, é necessário determinar normas e regras para que todos sigam: tanto os autores de material como os fabricantes de LMS. Uma dessas normas é o Sharable Content Object Reference Model (SCORM), aceita amplamente pelo mercado, conclusão que pode ser feita ao constatar que existem mais de 200 sistemas compatíveis (ADL, 2007), desde ferramentas de autoria, repositórios de conteúdos e LMS.

Esse artigo ilustra um dos recursos essenciais para o projeto instrucional que é o sequenciamento de atividades, e será demonstrado esse recurso dentro do modelo SCORM através de um curso produzido com o MOS Solo que é uma ferramenta livre de autoria de fácil utilização pelos professores, em que não é necessário ser profundo conhecedor do modelo SCORM. A produção do curso seguirá o modelo 4C/ID, exemplificando a necessidade de considerar métodos para melhorar a qualidade do material produzido.

\section{Sequenciamento do SCORM}

A especificação SCORM tem como objetivo promover o reuso de conteúdo instrucional (ADL, 2006b), através de regras que estruturam os arquivos com o conteúdo (assuntos, tarefas e exercícios) e o que um LMS deve fazer com esses arquivos, ou seja como interpretar e se comunicar com eles.

No SCORM o material instrucional deve ser no formato que possa ser apresentado num navegador web (browser). $\mathrm{O}$ arquivos com os conteúdos são compactados junto com outro arquivo que contém a estruturação desse conteúdo. Esse pacote de arquivos é denominado pacote de conteúdo. $\mathrm{O}$ arquivo com a estrutura do conteúdo é chamado de organização do conteúdo. Nesse arquivo são estipuladas as atividades de aprendizado e o sequenciamento das atividades.

Uma atividade pode ser um assunto, uma aula ou uma disciplina. Cada atividade de aprendizado é agrupada em um ou mais recursos independentes de outras atividades (ADL, 2006a). Um recurso pode ser uma página HTML, um arquivo de vídeo, áudio ou qualquer outro tipo que possa ser apresentado num navegador web. As atividades não podem apontar umas para as outras. O relacionamento entre elas é exclusivamente a sequência em que eles podem ser acessadas, e isso é estabelecido no arquivo da organização do conteúdo. Essa estratégia facilita a elaboração de novos cursos aproveitando atividades de cursos anteriores. As atividades podem conter conteúdo e tarefas para o aluno praticar, ou até ser uma avaliação.

O conteúdo é organizado de forma hierárquica: uma atividade pode conter subatividades, entretanto somente as atividades folhas (aquelas que não possuem subatividades) é que podem estar associadas aos recursos. Não é uma obrigação do SCORM, mas ele é fortemente baseado na idéia de existir visualmente uma árvore de atividades para que o aluno selecione diretamente aquela que lhe interessar ou precisar.

$\mathrm{O}$ sequenciamento é a possibilidade de criar regras para condicionar a apresentação das atividades (ADL, 2006c). Por exemplo, uma determinada atividade somente pode ser visível se outro conjunto de atividades for concluída, ou o aluno obteve no mínimo uma nota 6 num questionário. O SCORM estabelece as seguintes possibilidades de configuração do sequenciamento:

1. Controle de Requisição: como se comporta um grupo de atividades em relação às requisições de navegação do usuário. Uma requisição é a seleção de uma atividade 
que parte do usuário, seja clicando diretamente na atividade, ou pressionando um botão de próximo ou anterior. As configurações do controle de requisição determinam o fluxo de navegação (se é possível voltar uma atividade) e se pode selecionar uma atividade fora do conjunto de atividades que o usuário está interagindo;

2. Regras de Sequenciamento: é um conjunto de regras que podem ser usadas para habilitar/desabilitar ou mostrar/esconder uma atividade avaliando o estado das outras atividades. Por exemplo, uma atividade só é apresentada se uma outra atividade em específico foi concluída;

3. Restrições de Acesso: usado para limitar quantas vezes ou por quanto tempo uma atividade pode ser acessada;

4. Regras de Retropropagação: indica se o desempenho das subatividades refletem nas suas atividades de nível superior na árvore. Por exemplo, se uma atividade completa deve refletir que a atividade de nível acima também deve ser considerada completa;

5. Descrição de Objetivos: um objetivo é um campo especial da atividade para os recursos armazenarem informações. Por exemplo, para configurar na organização do conteúdo que uma atividade somente pode ser visível quando certa página de outra atividade for visitada, se faz o seguinte: cria-se um objetivo na atividade que contém a página a ser visitada, e nessa página programa-se para gravar algum valor no objetivo, e na atividade que depende dessa visita adicionam-se regras de sequenciamento para somente habilitá-la se o objetivo da outra atividade possuir um valor. As atividades podem ser primárias ou secundárias: as primárias são alimentadas automaticamente pelo LMS, com a mensuração do desempenho do aluno nos exercícios e com a informação se a atividade foi visitada por completa. Os objetivos secundários são aqueles em que os recursos podem gravar dados. Além disso, existem os objetivos locais e os globais. Os objetivos locais tem como escopo de visibilidade a própria atividade. Os recursos conseguem acessar somente esse tipo de objetivo. Para uma atividade acessar o objetivo de outra, esse objetivo precisa ser mapeado para um objetivo global. Nesse mapeamento configura-se a leitura e gravação de objetivos globais. No mapeamento de leitura determina-se o objetivo global a ser acessado e o nome do objetivo local destino. Na gravação é feita a relação de qual objetivo local é fonte para gravar os dados em qual objetivo global;

6. Controle de Aleatoriedade: com essas opções se configura para que o sistema possa selecionar aleatoriamente um conjunto de atividades.

Essas configurações de sequenciamento serão exemplificadas na seção 5 quando será apresentado um estudo de caso.

\section{Projeto Instrucional e Modelo 4C/ID}

Segundo Reigeluth (1999 apud Wiley, 2000), o projeto instrucional descreve métodos de como ensinar e as situações em que esses métodos devem ser aplicados. Um conteúdo a ser ensinado é dividido em pequenas partes, sequenciado e disponibilizado ao aluno de acordo com seu desempenho.

Existem várias teorias de projeto instrucional relatadas por Merrill (2002), tais

como: Star Legacy, 4-MAT, Instructional Episode, Multiple Approaches to Understanding, Collaborative Problem Solving, Constructivist Learning Environment, 4C/ID e Learning by Doing. De forma geral, Merrill identificou cinco princípios 
instrucionais que todos seguem, onde o aprendizado acontece quando: (i) os estudantes resolvem problemas do mundo real, (ii) o conhecimento existente é usado como fundamento para novo assunto, (iii) o novo conhecimento é demonstrado ao estudante, (iv) a nova matéria é aplicada pelo estudante, e (v) o novo assunto é integrado com o contexto do aluno.

Uma dessas técnicas relatadas por Merrill é o 4C/ID (Four Component Instructional Design). A forma de aprender nesse modelo é praticando, isto é, fazendo exercícios (Van Merriënboer e Kester, 2005). Eles são entregues inicialmente com bastante assistência (informações, exemplos, e guias oferecidos no próprio exercício) e progressivamente ela vai sendo eliminada das tarefas, até que o aluno tenha que resolver o problema sem qualquer ajuda. A demonstração dos conceitos e sua aplicação são integradas.

O conhecimento humano é estruturado na memória dividida duas partes: a memória de trabalho e memória de longa duração (Van Merriënboer e Kester, 2005; Clark e Lyons, 2004, p.24). As informações novas são processadas na memória de trabalho para transformarem-se em conhecimento e habilidades na memória de longa duração. A memória de trabalho suporta poucos elementos (informações) e por isso é muito limitada. É ali que ocorre o relacionamento entre esses elementos e com o conhecimento já consagrado. A memória de longa duração pode auxiliar na redução dessa limitação se as novas informações conseguirem ser relacionadas com $\mathrm{o}$ conhecimento dominado. A instrução é o resultado das transformações que acontecem na memória de trabalho integrada à de longa duração.

Esse modelo tem forte preocupação na redução da sobrecarga cognitiva. Essa redução acontece ao prover o máximo de ajuda necessária quando o estudante entra no novo assunto (menos informações sendo transferidas da memória de longa duração), e esse suporte vai reduzindo conforme o aluno avança nas tarefas (o conhecimento vai se formando na memória de longa duração).

Para aplicar o modelo 4C/ID, Van Merriënboer, Clark e Croock (2002) descrevem que é necessário dividir em pequenas partes o assunto pretendido ensinar ao aluno. Essas partes precisam ter um objetivo único e relacionadas às partes anteriores, criando uma sequência do mais simples, fundamental ou concreto, para o mais complexo, composto ou abstrato. Essa divisão resulta em aulas, e cada aula é estruturada nos quatro componentes do modelo 4C/ID, conforme ilustrado na figura 1.

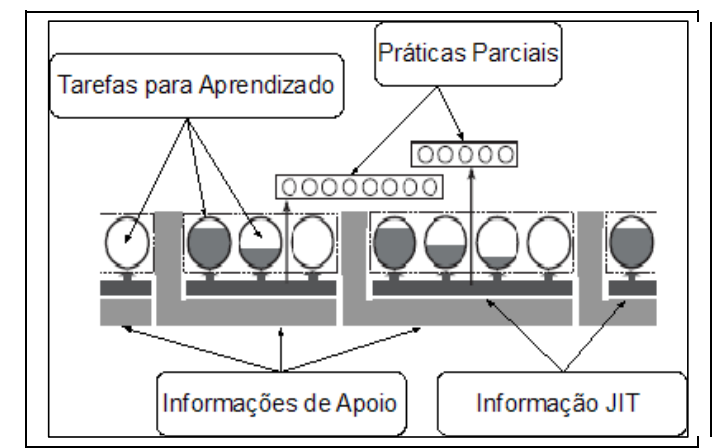

Figura 1 - Modelo 4C/ID

Adaptado de Van Merriënboer, Clark e Croock (2002)

Cada aula é formada por uma ou mais tarefas para o aprendizado. Elas são a prática em torno de um conceito e dentro de uma aula devem ser no mesmo nível de dificuldade. Entretanto, cada tarefa mostra casos ou situações diferentes sobre o conceito. Isso permite que a ordem de apresentação dessas tarefas seja sorteada. 
Contudo, a ordem de apresentação determina a quantidade de auxílio disponível para o aluno. A primeira com muito e a última sem nenhum apoio. Esse auxílio é a porção do exercício que vem pronto. A primeira tarefa é acompanhada com muitas respostas para as questões e a última é isenta de respostas. Cada tarefa deve ser composta por um estado inicial, um estado final aceitável, a solução, e o processo para a solução generalizada, isto é, como resolver qualquer problema desse tipo. O estado inicial representa uma situação inicial em um estudo de caso ou problema. O estado final é a resposta do problema. A solução é a sequência de operações que devem ser feitas partindo do estado inicial até alcançar o estado final. Porém, essas quatro partes não necessariamente são fornecidas ao aluno. De acordo com o nível de experiência dentro da aula, a tarefa pode exigir que o aluno formule um estado inicial dadas as outras partes, ou determine a sequência de passos dado um estado inicial e final. Isso serve para ilustrar como as tarefas não são simples questionários.

As informações de apoio são a teoria necessária para resolver os problemas. $\mathrm{Na}$ figura 1 é possível observar que esse componente aparece antes das tarefas de aprendizado, ou seja, essas informações são a primeira parte da aula. Esse é o momento de conectar novas informações com aquelas já praticadas pelo aluno. Depois essa informação acompanha as tarefas no mesmo nível. As informações de apoio podem ser modelos conceituais (o que é isso?), estruturais (como isso é organizado?) e causais (como isso funciona?). Sugere-se apresentar essas informações com exemplos e estudos de caso. A parte final da aula deve fornecer um retorno (feedback) ao aluno de como foi seu progresso através dos resultados das suas tarefas, comparando-os com de outros colegas ou com o previsto pelo projetista instrucional.

A informação JIT (Just-In-Time) refere-se a situações em que ocorrem várias vezes durante a instrução, independente da tarefa de aprendizado, e que são prérequisitos para executar a tarefa. Essa informação é representada por guias do tipo "como fazer" (how-to) e são acessadas pelo aluno para relembrar algum assunto. Por exemplo, numa aula sobre criar células de somatória em planilhas eletrônicas, o aluno precisa saber como se escrevem intervalos de células. Isso é um pré-requisito para criar esse tipo de fórmula, logo essa informação precisa estar disponível para acesso em todas as tarefas dessa aula. Entretanto, esse tipo de informação não representa ensinar a teoria por trás do conceito, mas um exemplo prático de como fazer, nesse caso, a seleção das células.

As práticas parciais também se referem às situações fundamentais que se repetem durante várias tarefas. Mas aqui, o objetivo é oferecer exercícios sobre esse tipo de informação, onde a mensuração do desempenho do aluno nessas práticas não interfere na mensuração do desempenho na aula. Na verdade, nem existe medida de desempenho nessas práticas parciais. Esses exercícios precisam ser simples, existirem vários, sobre assuntos já vistos por completo em aulas anteriores, e sempre estarem disponíveis para que o aluno reforce ou relembre algum conceito visto a algum tempo.

\section{Ferramenta MOS Solo}

A MindOnSite (www.mindonsite.com) possue três produtos voltados para o modelo SCORM:

- MOS Chorus: primeiro LMS compatível com SCORM 2004 3rd;

- MOS Solo: ferramenta de autoria de cursos;

- MOS Player: ambiente para execução dos cursos produzidos no MOS Solo. 
O MOS Solo é uma ferramenta de fácil utilização para produção de cursos. Ele possui recursos para uso de HTML para o autor produzir páginas de conteúdo e exercícios. Entretanto, os recursos em HTML são transparentes ao autor: é como se ele estivesse usando uma versão reduzida dos mais modernos processadores de texto. Existe disponível uma barra com ferramentas para formatação do texto, inclusão de imagens, links, tabelas e recursos multimídia como Flash, Windows Media, Real Player e QuickTime, em qualquer área em que se possa editar um conteúdo. Além disso, ele possui um editor de estilos, para que as páginas de todo o curso sigam automaticamente uma formatação, e a mudança no estilo se reflete em todo o curso.

Os cursos podem ser exportados como pacotes de conteúdo SCORM 2004, que é a versão mais atual dessa especificação. $O$ curso é gerenciado sobre uma árvore de atividades contendo elementos no modelo SCORM. Entretanto, o autor precisa conhecer algumas poucas regras sobre o funcionamento no modelo SCORM, mas essas regras são todas representadas por componentes de entradas (campos, caixas de verificação e seleção) na ferramenta, logo o autor precisa conhecer os recursos da ferramenta, e não diretamente os recursos do SCORM.

O MOS Solo categoriza quatro tipos de atividades: (i) a atividade de conteúdo, (ii) a atividade de avaliação, (iii) a atividade de treinamento e (iv) a sequência. $\mathrm{Na}$ ferramenta elas são chamadas de activitiy, assessment, questionnaire e sequence respectivamente.

A sequência é a única atividade que pode conter subatividades. Através desse tipo de elemento que se organiza e estrutura a árvore de atividades.

Os outros tipos de atividades são as únicas que podem conter as páginas. E essas páginas podem ser de conteúdo ou de exercícios. A diferença entre os três tipos de atividade é que as atividades de conteúdo não possuem qualquer tipo de mensuração no desempenho dos exercícios, as atividades de treinamento e avaliação guardam o desempenho do usuário no objetivo primário, mas a noção de aprovado acontece somente na atividade de avaliação.

Além das páginas de conteúdo, o MOS Solo dispõe de sete tipos de exercícios que podem ser incorporadas às atividades.

Cada tipo de página possuem um editor específico. Os editores de exercícios possuem recursos para que o autor insira dicas ao usuário, configure os textos das mensagens de sucesso ou erro, direcionamento para outras páginas segundo condições de sucesso ou fracasso.

As configurações de sequenciamento apresentadas na seção 2 estão disponíveis nos quatro tipos de atividades, entretanto, existem opções disponíveis diferentes em cada um dos tipos, mesmo porque existe essa diferença no próprio SCORM: atividades que possuem outras atividades tem opções diferentes das atividades folhas.

Apesar da quantidade de opções disponíveis na ferramenta, pois são várias as possibilidades no modelo SCORM, é possível clicar nas opções para obter uma breve explicação sobre ela, o que auxilia muito na usabilidade da ferramenta.

O MOS Solo, além de fácil ferramenta de autoria para professores, é excelente ferramenta para laboratório no estudo de sequenciamento das atividades, pois ao editar o curso no mesmo instante já é possível testá-lo. Todas as opções do SCORM foram implementadas, logo é possível fazer um estudo completo sobre o assunto. 


\section{Estudo de Caso}

Para demonstrar o uso do MOS Solo com os recursos de sequenciamento do SCORM e aplicação do modelo 4C/ID, foi desenvolvido um curso para ensino das regras básicas de Xadrez baseado em Fontarnau (2003) e Girona et al (2003).

$\mathrm{Na}$ metodologia com o 4C/ID, a primeira atividade é a identificação e particionamento dos assuntos para que cada parte se refira a um conceito bem específico e limitado. Baseado nos autores citados no parágrafo anterior, o curso foi estruturado conforme ilustra a figura 2. Essa figura apresenta o curso de forma abreviada, limitando para o que será usado nessa seção. A aula a ser produzida será sobre movimentação de peões.

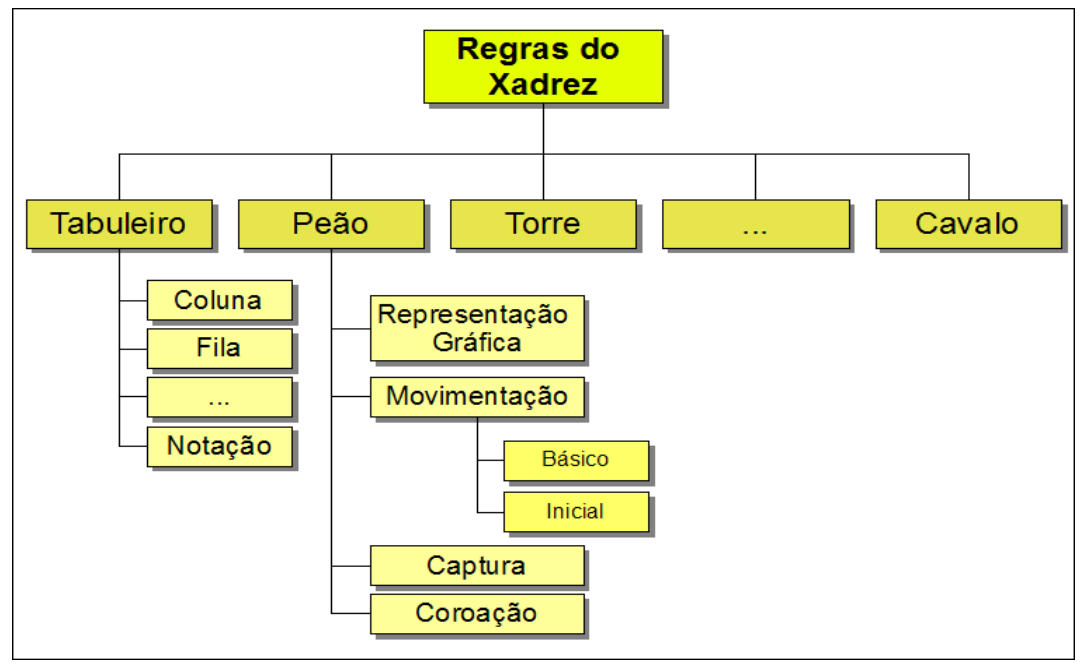

Figura 2 - Estrutura do conteúdo do curso "Regras do Xadrez"

Pode-se observar que o tabuleiro é visto antes das peças do jogo, e que as peças são ensinadas individualmente. A estrutura apresentada para o peão é parecida para as demais peças: representação gráfica, movimentação e captura acontecem em todas elas.

Essa árvore é importante para que o projetista saiba o que já foi ensinado a certa altura do curso. Por exemplo, quando forem desenvolvidas as atividades sobre peões, deve-se considerar que o aluno já saiba tudo sobre tabuleiro. Mas, seguindo o modelo 4C/ID, recomenda-se que o assunto sobre tabuleiros possa ser disponibilizado ao aluno na forma de informações JIT ou práticas parciais, no momento que tais conceitos forem necessários para executar alguma tarefa.

O modelo 4C/ID indica para que as tarefas de aprendizado precisam ser todas de mesmo nível de dificuldade, e diferenciarem-se pela quantidade de suporte ao aluno, ou seja, a quantidade respostas já inclusas no próprio exercício. As tarefas poderiam inclusive serem sorteadas e o sistema prover a quantidade de suporte de acordo com a atividade em que o aluno se encontra. Para que isso seja possível nas páginas, precisaria ser programado nos próprios recursos para que eles soubessem a ordem da tarefa em que eles se encontram, ou o nível de experiência, e decidir se mostra ou não a resposta para uma determinada parte do conteúdo. Porém, o MOS Solo não possui recursos nesse nível. Por essa razão, as tarefas de aprendizado foram produzidas já com a quantidade de suporte embutidas nelas.

A figura 3 apresenta como as atividades foram estruturadas seguindo o modelo 4C/ID e pensando na produção com o MOS Solo. "Movimentação" representa a aula no 
modelo e foi criada como sequência na ferramenta. O nó "Teoria" foi criado como atividade de conteúdo e os demais foram criados como atividades de avaliação.

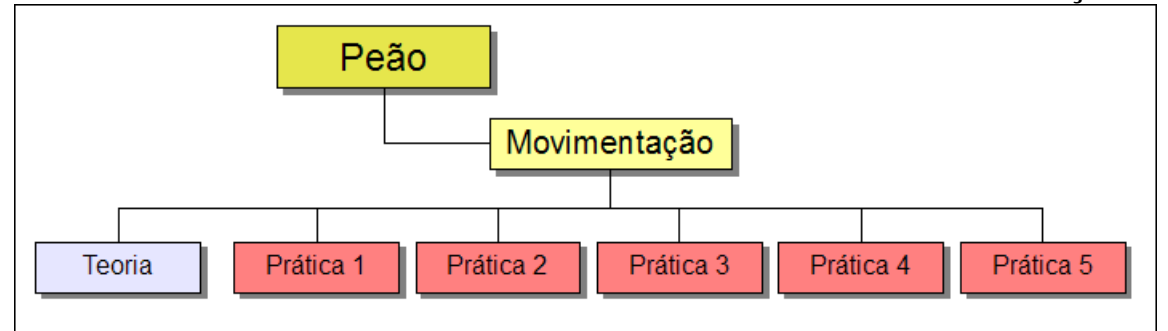

Figura 3 - Estrutura da aula "Movimentação"

A primeira atividade é a informação de apoio, e conforme o modelo 4C/ID, ela deve apresentar o assunto a ser abordado na aula, e relacionar com os assuntos vistos anteriormente. Nessa atividade é relembrada a posição dos peões no começo do jogo, qual o movimento do peão, um alerta sobre o peão não poder recuar, e o movimento especial que o peão pode fazer logo no começo. Foi criada uma página para cada um desses conceitos, contendo texto, imagem e animação Flash.

As práticas representam as tarefas de aprendizado e foram desenvolvidas para que a tarefa seguinte somente possa ser acessível se a anterior foi concluída, mesmo com fracasso. Para cada prática foram criadas três páginas de exercícios e o LMS sorteia uma delas. Foram idealizadas três categorias de exercícios: peões que podem se movimentar, peões que não podem se movimentar e movimentar os peões. Na primeira categoria o aluno precisa selecionar quais são os peões que podem se movimentar. $\mathrm{Na}$ segunda quais são os que não podem e na última ele vai movimentar os peões.

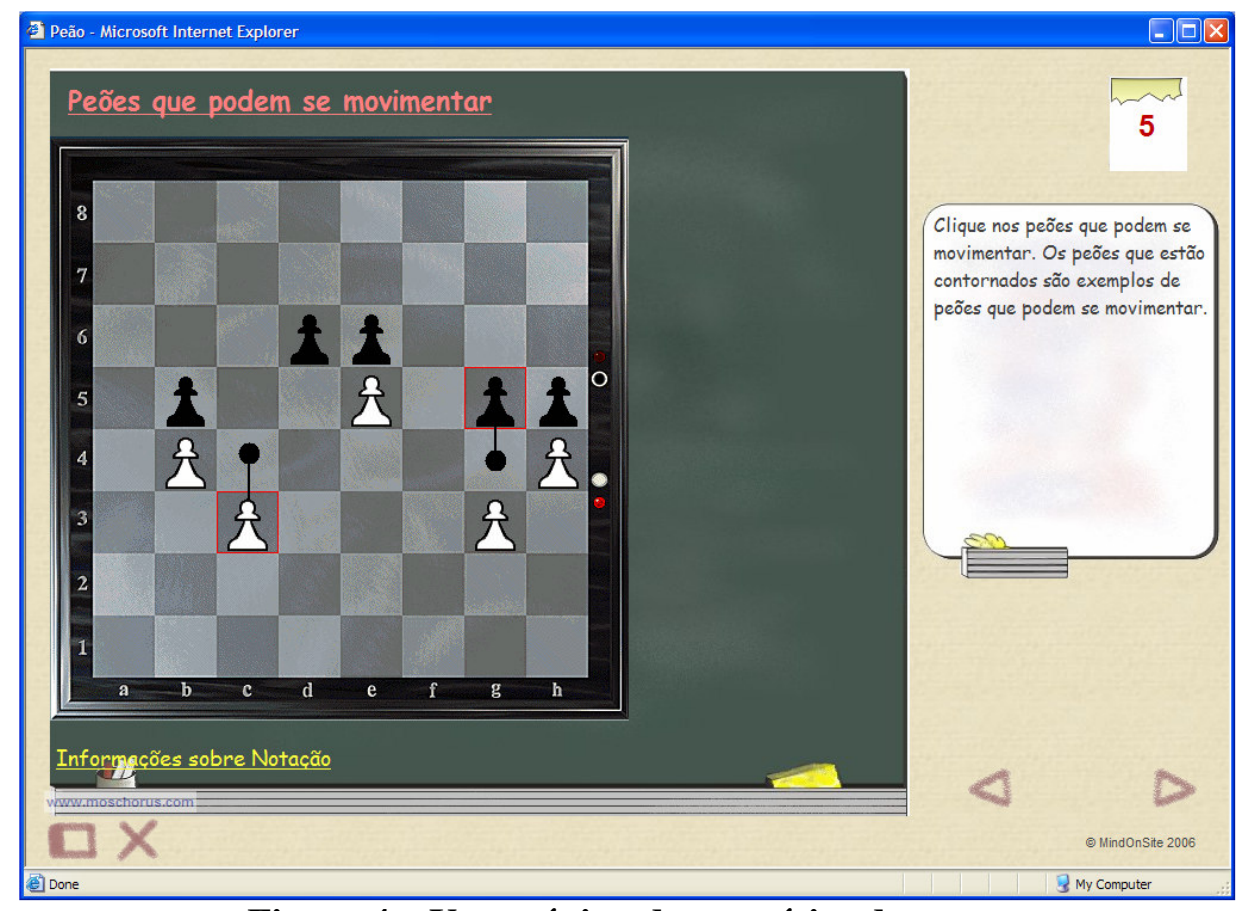

Figura 4 - Uma página de exercícios do curso

A "prática 1" tem páginas sobre a primeira categoria, a "prática 2" sobre a segunda e a "prática 3" sobre a terceira categoria. Todas essas páginas possuem bastante auxílio ao estudante, apresentando na própria página alguns peões já marcados. $\mathrm{Na}$ 
prática 4 foram criados três exercícios um para cada uma das três categorias mas com metade do auxílio oferecidos pelas práticas anteriores. Os exercícios da prática 5 não tem ajuda alguma ao usuário ele deve resolvê-las sozinho. Para cada uma das tarefas de aprendizado é oferecido auxílio sobre notações, através de informações JIT e práticas parciais. Esse auxílio pode ser acessado através de um link nas páginas. Em alguns exercícios o aluno utiliza o mouse e são apresentadas as notações sobre o movimento da peça, e em outros exercícios o aluno precisa digitar na notação os movimentos a serem executados. A figura 4 ilustra uma das páginas de exercício onde o aluno precisa clicar nos peões que podem se movimentar. No final da página existe um link "Informações sobre Notação" para acessar as informações JIT e práticas parciais.

Para implementar as condições para uma prática ficar disponível somente se as anteriores foram concluídas, utilizaram-se objetivos e regras de sequenciamento. Em cada uma das atividades foi criado um objetivo primário e mapeado para gravação como objetivo global. Por exemplo, "na prática 1" foi criado o objetivo primário "obj_pratica1" e mapeado num objetivo global de mesmo nome. Também foram adicionados objetivos secundários mapeados para leitura dos objetivos globais da prática anterior. Seguindo o exemplo, na "prática 2" foi adicionado um objetivo secundário "obj_pratica1" e mapeado para ler do objetivo global "obj_pratica1".

$\mathrm{Na}$ "prática 2" em diante, foram adicionadas duas regras de sequenciamento para que ela fique desabilitada enquanto a anterior não for satisfeita. Ambas as regras fazem uma comparação com o objetivo secundário "obj_pratica1". A primeira regra desabilita o item enquanto o valor do objetivo for desconhecido, e a segunda desabilita o item enquanto o valor do objetivo não for satisfeito. O SCORM determina que se um valor é desconhecido, isto é, se jamais foi gravado um valor nele, qualquer comparação que se faça sempre retornar como verdadeiro. Daí a necessidade de adicionar as duas regras.

$\mathrm{Na}$ ferramenta foram criadas atividades adicionais para as informações JIT e práticas parciais. Porém, elas foram configuradas para não estarem disponíveis no curso, e com isso, não haver a possibilidade de acessá-las através da árvore de atividades. Nas páginas das tarefas foram adicionados hiperlinks para as páginas dessas atividades, e configuradas para que essas páginas sejam abertas numa nova janela.

\section{Conclusões}

O MOS Solo tem um grande apelo pela facilidade de usuários sem conhecimento técnico de webdesign poderem produzir páginas de conteúdo. Além disso, com ela se conseguem criar sete formas diferentes de exercícios e atividades práticas para o aluno. A inserção de links, edição de imagens e conteúdos foi bastante natural, sem exigência de programação com HTML. Os editores de exercícios são poderosos, permitindo criar caminhos de páginas dependente e dicas de acordo com o sucesso ou fracasso na execução do exercício.

O sequenciamento exige um conhecimento básico, mas a configuração dele se dá através de caixas de verificação ou formulários com poucos campos e bem esclarecidos. A todo instante é possível acessar uma explicação sobre cada campo ou seção de campos.

No estudo de caso desse artigo, a natureza dos exercícios de xadrez exige flexibilidade para movimentar peças e avaliar a configuração do tabuleiro. A criação dos exercícios consistia de importar a imagem do tabuleiro desenhada com ou sem peões, e importar as imagens dos peões. O MOS Solo permitiu que a maioria dos exercícios fossem elaborados com facilidade, porém, os exercícios de movimentação 
ficaram limitados quanto ao retorno para o aluno. Num tabuleiro com um conjunto de peões, não foi possível configurar para que o LMS informasse qual dos peões foi movimentado incorretamente. $\mathrm{O}$ sistema avalia somente se o exercício está ou não correto.

$\mathrm{Na}$ adoção do modelo 4C/ID foi necessário quebrar uma das regras básicas do SCORM: uma atividade não pode se relacionar a outra. Isso aconteceu no momento de oferecer informações JIT e práticas parciais sobre notação. Todas as práticas tem acesso a esses componentes, e são exatamente os mesmos. O SCORM permite que uma mesma página pertença a mais de duas atividades, mas a ferramenta não dispõe desse recurso. Por essa razão, foi criada uma atividade exclusivamente para esses componentes, que puderam ser eliminadas da estrutura de árvore de atividades, diminuindo o impacto na quebra desse regra.

\section{Referências}

ADL. Sharable Content Object Reference Model - SCORM 2004 2nd Edition - Content Aggregation Model. Novembro, 2006a.

Sharable Content Object Reference Model - SCORM 2004 2nd Edition - Overview. Novembro, 2006b.

. Sharable Content Object Reference Model - SCORM 2004 2nd Edition - Sequencing and Navigation. Novembro, 2006c.

Certified Products. Disponível em: <http://www.adlnet.gov/scorm/certified/index.aspx> Acesso em: 24 out. 2007.

CLARK,R.C.; LYONS,C.Graphics for Learning.San Francisco:John Wiley\&Sons,2004.

FONTARNAU, A.S. O ensino de xadrez na escola. Porto Alegre: Artmed, 2003.

GIRONA, P.C.; POLLAN, O.B.; COUBA, C.C.; GIRONA, M.J.C. Iniciação ao xadrez para crianças. Porto Alegre: Artmed, 2003.

IRLBECK, S. MOWAT, J. Learning Content Management Systems (LCMS). In: Learning Objects: Standards, Metadata, Repositories \& LCMS. Keith Harman e Alex Koohang (eds). California: Informing Science Press, 2007.

MERRIL, M. D. First Principles of Instruction. In:ETR\&D.Vol.50. No.3,2002, p.43-59.

TAROUCO, L.M.R.; KONRATH, M.L.P.; CARVALHO, M.J.S.; AVILA, B.G. Formação de professores para produção e uso de objetos de aprendizagem. RENOTE - Revista Novas Tecnologias na Educação: IV Ciclo de Palestras sobre Novas Tecnologias na Educação. Porto Alegres, RS, 2006.

van MERRIËNBOER, J.J.G.; CLARK, R.E.; CROOCK, M.B.M. Blueprints for Complex Learning: The 4C/ID-Model. In: ETR\&D. Vol. 50. No. 2, 2002, p. 39-64.

van MERRIËNBOER, J.J.G.; KESTER, L. The Four-Component Instructional Design Model: Multimedia Principles in Environments for Complex Learning. In R.E.Mayer (Ed.), The Cambridge Handbook of Multimedia Learning. New York: Cambridge University Press, 2005.

WILEY, D. A. Learning Object Design and Sequencing Theory. 2000. 